\title{
Efecto del muestreo de diferentes hábitats sobre la aplicación de índices bióticos basados en macroinvertebrados
}

\author{
Marta Leiva ${ }^{1}$, ERnestina Bonacina ${ }^{2}$ y Liliana Diodato 3 \\ 1 UNSE-CONICET. Instituto de Protección Vegetal, Facultad de Ciencias Forestales, Universidad Nacional de \\ Santiago del Estero (INPROVE FCF UNSE). Avda. Belgrano No 1912 Santiago del Estero, Argentina CP 4200 \\ 2 UNSE-CONICET. Instituto de Silvicultura y Manejo de Bosques, Facultad de Ciencias Forestales, Universidad \\ Nacional de Santiago del Estero (INSIMA FCF UNSE) \\ 3 UNSE. Instituto de Protección Vegetal, Facultad de Ciencias Forestales, Universidad Nacional de Santiago \\ del Estero \\ E-mail: martaleiva@unse.edu.ar
}

\begin{abstract}
RESUMEN. El objetivo de este estudio fue evaluar el efecto del hábitat en la aplicación de índices bióticos (BI) basados en macroinvertebrados bentónicos. Se muestrearon dos hábitats, (macrófitas y sedimentos), en dos sitios del río Dulce (Cuenca Salí-Dulce, Argentina): S1 (27 $31^{\prime}$ $\left.5.82^{\prime \prime} \mathrm{S}, 64^{\circ} 52^{\prime} 41.37^{\prime \prime} \mathrm{O}\right)$ y S2 ( $\left.27^{\circ} 28^{\prime} 36.21^{\prime \prime} \mathrm{S}, 64^{\circ} 49^{\prime} 30.30^{\prime \prime} \mathrm{O}\right)$. El muestreo se realizó con caudal bajo, octubre/2015. Se midió $\mathrm{pH}$, conductividad eléctrica $(\mathrm{CE} \mu \mathrm{S} / \mathrm{cm})$, temperatura $\left(\mathrm{T}^{\circ} \mathrm{C}\right)$, sólidos suspendidos totales (SST mg/L), oxígeno disuelto (OD mg/L) y demanda biológica de oxígeno $\left(\mathrm{DBO}_{5}\right.$ $\mathrm{mg} / \mathrm{L}$ ). Los macroinvertebrados se filtraron con red de nylon de $250 \mu \mathrm{m}$ y se identificaron al nivel taxonómico requerido para la aplicación de los IB. Se analizaron riqueza taxonómica y diversidad. Se aplicaron tres IB: el IBMWP, el BMWP' adaptado al río Salí y el IMRP desarrollado para la ecoregión Pampa de Argentina. También se aplicó el ASPT asociado con los BMWP'. Los parámetros físicos y químicos mostraron buena calidad del agua, con un $\mathrm{pH}$ promedio de 8,57; CE $773 \mu \mathrm{S} / \mathrm{cm} ; \mathrm{T} 19,5^{\circ} \mathrm{C}$; SST 9,27 mg/L; OD 7,49 mg/L y DBO 53,29 mg/L. Los taxones registrados fueron 34 y la diversidad fue mayor en las macrófitas que en los sedimentos. Los IB mostraron resultados diferentes según el hábitat donde se aplicaron. Las macrófitas contribuyeron con el doble de taxones a los IB, en comparación con los sedimentos. Los valores de ASPT correspondieron a calidad del agua impactada. El IMRP fue el más inclusivo en cantidad de taxones para el cálculo. La conclusión es que el tipo de hábitats influye en la aplicación de los IB.
\end{abstract}

Palabras clave: Biomonitoreo, calidad de aguas, cuenca Salí-Dulce.

ABSTRACT. The aim of this study was to evaluate the effect of habitat on the application of biotic indices (BI) based on benthic macroinvertebrates. Two different habitats were sampled, (macrophytes patches and sediments), in two sites in the Dulce River (Salí-Dulce basin, Argentina): S1

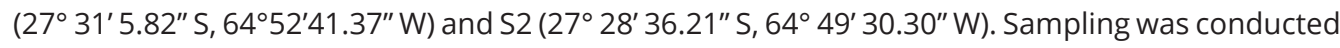
in a low-flow period, 2015-October. The variables measured were $\mathrm{pH}$, electric conductivity (EC $\mu \mathrm{S} / \mathrm{cm}$ ), temperature $\left(\mathrm{T}^{\circ} \mathrm{C}\right.$ ), total suspended solids (TSS $\mathrm{mg} / \mathrm{L}$ ), dissolved oxygen (OD $\mathrm{mg} / \mathrm{L}$ ) and biological oxygen demand $\left(\mathrm{BOD}_{5} \mathrm{mg} / \mathrm{L}\right)$. Macroinvertebrates were filtered with a nylon net of 250 $\mu \mathrm{m}$ pore size and were identified at the required taxonomic level for BI application. Taxonomic richness and diversity were analyzed. Three BI were applied: the IBMWP for the Iberian Peninsula, the BMWP' adapted for Salí River basin and the IMRP developed for the Pampean ecoregion from Argentina. Also score ASPT associated with BMWP' was applied. Physical and chemical parameters showed good water quality, with a pH average of 8.57; EC $773 \mu \mathrm{S} / \mathrm{cm}$; T 19.5 ${ }^{\circ} \mathrm{C}$; TSS $9.27 \mathrm{mg} / \mathrm{L}$; $\mathrm{DO} 7.49 \mathrm{mg} / \mathrm{L}$; and $\mathrm{BOD}_{5} 3.29 \mathrm{mg} / \mathrm{L}$. The registered taxa were 34 and the diversity was greater in macrophytes than in sediments. The BI showed different results according to the habitat where 
they were applied. Macrophytes contribute with twice of the taxa for the BI calculations, compared with sediments. The ASPT values corresponded to impacted water quality. The IMRP was the most inclusive in terms of the number of taxa for calculation. The conclusion is that the type of habitats influences the BI application.

Keywords: Biomonitoring, Salí-Dulce basin, water quality.

\section{INTRODUCCIÓN}

Los ecosistemas acuáticos continentales se cuentan entre los más amenazados del planeta, y esta situación se incrementa sostenidamente (Reid et al., 2019). La necesidad de conservación de los ecosistemas acuáticos y de mantener la sostenibilidad de los servicios que prestan al desarrollo de la vida y de las actividades humanas lleva a conservar intacta la preocupación por el monitoreo de su calidad ecológica. La calidad definida por indicadores bióticos es una metodología ampliamente utilizada para el monitoreo de agua, ya que complementando a los parámetros de calidad física y química ofrece una visión integral de la situación del ecosistema evaluado (Prat-Fornells et al., 2009; Roldán-Pérez, 2016; Segnini, 2003).

Los índices bióticos (IB) son ampliamente utilizados como herramientas concretas de evaluación de calidad de aguas. Estos índices otorgan un valor numérico según el nivel de tolerancia o sensibilidad que diferentes grupos de taxones tienen a la contaminación. Entre ellos, los formulados en base a familias de macroinvertebrados bentónicos pueden ser considerados los más ampliamente aplicados (Bonada et al., 2006; Prat-Fornells et al., 2009). Los macroinvertebrados bentónicos son los invertebrados que viven en los cuerpos de agua asociados al sedimento, a la vegetación acuática o a diversos elementos que pueden utilizar como sustrato de apoyo y hábitat, y que además pueden verse a simple vista o son retenidos por una malla de $500 \mu \mathrm{m}$ (Hanson et al., 2010), aunque de acuerdo con las regiones de trabajo y características de los hábitats muestreados algunos autores recomiendan el uso de redes de menor abertura (Domínguez y Fernández, 2009) y en los estudios ecológicos es común el uso de redes de $250 \mu \mathrm{m}$ de abertura de malla (Ramírez, 2010). (Bonada et al.,
2006) destaca una serie de ventajas que los hacen aptos como bioindicadores, entre éstas, su ocurrencia en prácticamente todos los ecosistemas acuáticos; su gran riqueza de especies, que ofrece un espectro muy amplio de respuestas; su carácter relativamente sedentario, lo que facilita el análisis espacial de los efectos de la contaminación; la propensión de ciertas especies a entrar en la columna de agua (deriva), que puede indicar la presencia de un contaminante; la larga vida de algunas especies, que pueden utilizarse para rastrear efectos a lo largo de diferentes períodos de tiempo; su compatibilidad con equipos de muestreo económicos; una taxonomía para géneros y familias bien conocida y en constante desarrollo; la sensibilidad de muchas especies para diferentes tipos de contaminación y su idoneidad para estudios experimentales.

Actualmente existen diversos IB formulados con base en macroinvertebrados bentónicos. Los más populares son los basados en el método BMWP (Biological Monitoring Working Party) diseñado originalmente para ríos de Inglaterra (Armitage et al., 1983). Este índice se obtiene a partir de la sumatoria de los valores de sensibilidad de cada una de las familias registradas en el ecosistema, dando como resultado un valor del índice que representa una clase de calidad del agua. El valor del indicador aumenta cuantas más familias sensibles a la contaminación se encuentren en el sitio evaluado. Asociado a este índice se calcula el ASPT (Average Score Per Taxon) obtenido a partir de dividir el valor final del índice BMWP por el número de familias incluidas en el cálculo de este, de manera que el ASPT representa el valor medio de tolerancia de la comunidad. A partir del BMWP original se desarrollaron numerosos ajustes alrededor del mundo, adaptando este popular índice a diferentes ecorregiones y sistemas fluviales. Entre las más 
reconocidas de estas adaptaciones se encuentra el IBMWP adaptado para los ríos de la Península Ibérica por Alba-Tercedor y Sánchez-Ortega (1988). En la Argentina, este mismo índice fue ajustado a la cuenca del río Salí por Domínguez y Fernández (1998). Un índice biótico similar, el IMRP (Índice de Macroinvertebrados para Ríos Pampeanos) fue diseñado por Rodrigues Capítulo et al. (2003) para los arroyos de la ecorregión Pampa de la Argentina. En general, los protocolos de aplicación de IB plantean la realización de muestreos multihábitat utilizando redes que pueden recolectar los invertebrados asociados a diferentes sustratos (Barbour, et al., 1999; Jáimez-Cuéllar et al., 2002; Domínguez y Fernández, 2009). Cuando se trabaja con los invertebrados asociados a un sustrato en particular y con muestreadores específicos para dicho sustrato, como puede ser el sedimento de fondo o los parches de macrófitas, se corre el riesgo de perder información por omisión de taxones asociados a otros hábitats. Macchi (2016), comprueba en humedales de la ecoregión de la Patagonia Argentina, que el muestreo de diferentes hábitats, particularmente macrófitas en columna de agua, por un lado, y sedimentos por otra parte, con diferentes métodos de muestreo respectivamente, aportan diferentes grupos de taxones a la biodiversidad del ecosistema estudiado. Estos taxones mostraron especificidad por los ambientes analizados, por lo que se recomienda que para la aplicación de herramientas de biomonitoreo, cuando éstas son influenciadas por los valores de biodiversidad del sistema, resulta deseable cubrir todos los hábitats disponibles.

Sin embargo, conocer hasta qué punto el tipo de muestreo que se realiza influye sobre la posible aplicación de herramientas de biomonitoreo no resulta tan simple en ecosistemas de gran extensión y poco estudiados. En ríos de mayor tamaño pueden resultar más laboriosas las tareas de muestreo, volviendo la práctica del biomonitoreo menos expeditiva. Clarificar cuánto influyen los hábitats disponibles sobre la diversidad y la riqueza de macroinvertebrados y cómo afectan la evaluación de calidad de aguas mediada por índices bióticos resulta de interés para ajustar las herramientas de biomonitoreo a los sistemas ecológicos bajo estudio.
La información sobre la diversidad de macroinvertebrados acuáticos en el río Dulce es aún incipiente. El uso de herramientas de biomonitoreo en este sistema fluvial resulta novedoso y requiere profundizar en la generación de experiencias a fin de ajustar su aplicación a las características locales. En este trabajo, se caracteriza la diversidad de familias de invertebrados acuáticos asociados a dos tipos de soportes en el hábitat fluvial: las macrófitas y los sedimentos de fondo; a la vez que se aplican índices bióticos de calidad de agua para ambos hábitats por separado y en conjunto. El objetivo fue evaluar el efecto de diferentes hábitats sobre la aplicación de IB en este sistema fluvial en particular.

\section{MATERIALES Y MÉTODOS Área de estudio}

El estudio se llevó a cabo en el río Dulce, tramo medio de la cuenca endorreica Salí-Dulce, la cual nace en las provincias de Salta y Catamarca, y pasando por Tucumán y Santiago del Estero, vuelca sus aguas en la Laguna Mar Chiquita, en la provincia de Córdoba. Desde el año 1963 el Dulce es un río regulado por la presa de Río Hondo, y mantiene su pulso de crecida en los meses de enero a marzo con caudales estables a lo largo del resto del año, de abril a diciembre. Se ubica en la ecorregión del Chaco Seco (Burkart et al., 1999; Brown y Pacheco, 2006) con clima semiárido, temperatura media anual variando de Norte a Sur entre $23^{\circ} \mathrm{C}$ a $18^{\circ} \mathrm{C}$ y precipitaciones marcadamente estivales, entre los meses de octubre a marzo, con valores anuales de 500 a $700 \mathrm{~mm}$, en un gradiente de disminución con sentido Este a Oeste.

\section{Muestreos}

Se establecieron dos sitios sobre la ribera derecha, en temporada de aguas bajas, durante el mes de octubre del año 2015. El

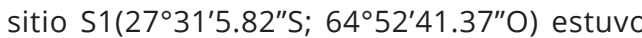
localizado al frente de la Reserva Natural Urbana Tara Inti. El lecho del río en este sitio 
es rocoso, con acumulación de arena en las zonas deposicionales de la ribera y en las islas. Sobre las riberas se observó desarrollo de macrófitas arraigadas y flotantes. El sitio S2 ( $27^{\circ} 28^{\prime} 36.21^{\prime \prime} \mathrm{S}, 64^{\circ} 49^{\prime} 30.30^{\prime \prime}$ O) se localiza aguas abajo de la ciudad de Termas de Río Hondo, a una distancia de aproximadamente $8 \mathrm{~km}$, medidas por el curso de agua (Figura 1). Este sitio se caracteriza por la presencia

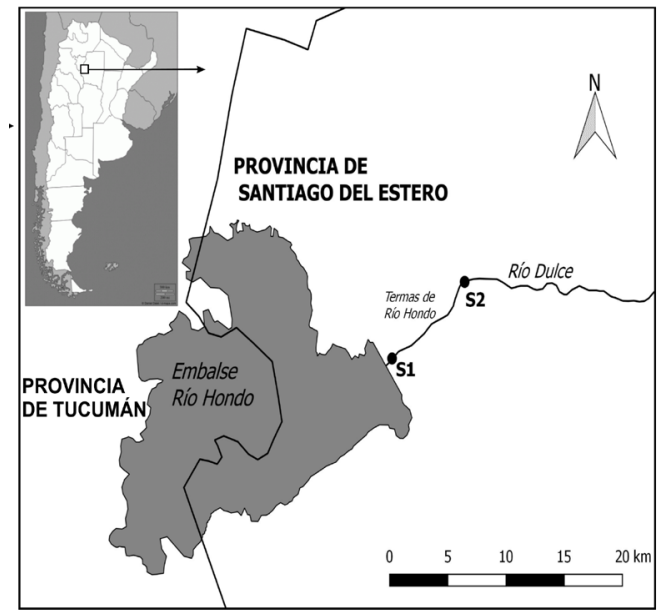

Figura 1. Localización del área de estudio y los sitios de muestreo.

Figure 1. Study area and sampling site locations.

de un área de remanso, en continuidad a un tramo de rápidos escalonados formados por afloramientos de arcillas que atraviesan el lecho del río. En esta área de remanso se desarrollan principalmente macrófitas flotantes. Para el muestreo, se seleccionaron dos hábitats en cada sitio: los parches de macrófitas y las áreas de depositación de sedimentos en cercanías a los parches de macrófitas, con el interés de conocer y comparar cuál es la diversidad asociada a los mismos en el río Dulce. Se tomaron las muestras dentro de una franja de $5 \mathrm{~m}$ desde la margen del río hacia el interior del lecho. La presencia y características de las macrófitas se evaluaron sólo cualitativamente. No se realizó una identificación a nivel de especies y tampoco se analizó cuantitativamente su diversidad. El S1 presentó un banco de macrófitas palustres conformado principalmente por especies de la familia Cyperaceae entre las cuales se desarrollaban otras macrófitas flotantes, pertenecientes a los géneros Eichhornia,
Ceratophyllum, Azolla y Lemna. El S2 presentó un parche de macrófitas flotantes desarrollado en un área de remanso sobre la ribera del río, con predominio de los géneros Eichhornia y Pistia.

\section{Muestreo de invertebrados en macrófitas}

Se realizó un muestreo cualitativo utilizando una red de mano cuadrangular de $30 \times 30$ cm, unida a un mango de 1,5 $\mathrm{m}$ de largo, con copo de nylon de $250 \mu \mathrm{m}$ de abertura de malla. La red se sumergió y retiró dos veces consecutivas, y se sumaron ambas colectas para conformar la muestra. Las plantas de mayor tamaño que quedaron atrapadas en la red se enjuagaron en el lugar con agua del río, dentro de la red para recolectar los organismos que pudieran desprenderse de ellas. Los macroinvertebrados, junto con restos vegetales y plantas más pequeñas, atrapados en la red fueron guardados en frascos plásticos de $1 \mathrm{~L}$ de capacidad y fijados con formol al 5\% vol.

\section{Muestreo de invertebrados en sedimentos}

El muestreo de invertebrados asociados al sedimento se realizó utilizando un muestreador tipo Core de $10 \mathrm{~cm}$ de diámetro. Se localizó el punto de muestreo al lado del parche de macrófitas, pero en hábitat de agua libre sin vegetación, en la zona de depósito de la ribera. Se introdujo el muestreador hasta los $10 \mathrm{~cm}$ de profundidad y se tomaron tres unidades muestrales en cada sitio para disminuir el efecto de parche que suelen presentar los ensambles de invertebrados bentónicos, separando las unidades muestrales una distancia aproximada de $1 \mathrm{~m}$ entre cada una de ellas y evitando disturbar el sedimento previo a la toma de la muestra. Estas unidades muestrales fueron sumadas conformando una sola muestra por sitio, y se analizó el total de organismos recolectados. El sedimento recogido fue filtrado a través de un tamiz de nylon de 250 $\mu \mathrm{m}$ de abertura de malla. El sedimento con los invertebrados retenidos en el tamiz fue guardado en frascos plásticos con formol al $5 \%$ vol. 


\section{Medición de variables físico-químicas}

En los sitios se midió $\mathrm{pH}$, conductividad eléctrica $(\mathrm{CE} \mu \mathrm{S} / \mathrm{cm})$ y temperatura $\left(\mathrm{T}^{\circ} \mathrm{C}\right)$ con medidores digitales. Se tomaron muestras para análisis de sólidos suspendidos totales (SST mg/L), por el método gravimétrico; oxígeno disuelto (OD $\mathrm{mg} / \mathrm{L}$ ) por método Winkler y demanda biológica de oxígeno $\left(\mathrm{DBO}_{5} \mathrm{mg} / \mathrm{L}\right)$ por incubación a $20^{\circ} \mathrm{C}$ en 5 días, siguiendo las técnicas normalizadas por APHA (1989). Se midió la profundidad (cm) en el sitio de muestreo con una vara graduada y la velocidad (en $\mathrm{m} / \mathrm{s}$ ) se estimó utilizando naranjas como flotadores en una distancia de $5 \mathrm{~m}$ con tres repeticiones. En conjunto con las muestras de invertebrados asociados a sedimentos se tomó una muestra para determinar la composición granulométrica. Ésta se analizó mediante el método de tamizado húmedo (Gee and Bauder, 1986), separando las fracciones de cantos ( $>20 \mathrm{~mm}$ ), gravas $(>2 \mathrm{~mm})$, arenas gruesas $(>0,63 \mathrm{~mm})$, arenas medias $(>0,25 \mathrm{~mm})$, arenas finas $(>0,063 \mathrm{~mm}) \mathrm{y}$ limos-arcillas $(<0,063 \mathrm{~mm})$.

\section{Procesamiento de muestras de invertebrados y análisis de datos}

En laboratorio se separaron manualmente los invertebrados del material vegetal y de los sedimentos bajo lupa estereoscópica. Los organismos limpios fueron guardados en alcohol $70^{\circ}$ y se identificaron hasta los niveles taxonómicos procurados para la aplicación de los índices bióticos seleccionados, siguiendo las claves taxonómicas de Domínguez y Fernández (2009) y Hamada et al. (2014). Se calculó la riqueza taxonómica y el índice de ShannonWiener $\left(\mathrm{H}^{\prime}\right)$ con el programa estadístico PAST versión 3 (Hammer et al., 2018). Se aplicaron Ios IB IBMWP de Alba-Tercedor y SanchezOrtega (1988), desarrollado para la Península Ibérica y el BMWP' adaptado para la Cuenca del Salí por Domínguez y Fernández (1998). Para las versiones del BMWP' se calculó el scord asociado ASPT (Armitage et al., 1983). Se aplicó también el IMRP (Rodrigues Capítulo et al., 2003), desarrollado para la ecorregión Pampa de la Argentina. Los protocolos de aplicación de los IB así como los valores de sensibilidad que éstos asignan a los taxones se pueden consultar en las fuentes bibliográficas citadas. Los IB se aplicaron sin realizar modificaciones en los valores de sensibilidad de los taxones. Tampoco se asignaron valores a taxones registrados que no contaran con un valor en el protocolo de los IB. En el caso de Odonata, se asignó el valor considerando la opción que otorga valor 8 a "Odonata (varias familias)" en el BMWP' Salí, mientras en el IMRP se aplicó el valor medio dentro del rango 1,2-1,6 propuesto para "Odonata (larvas)" en las tablas de dicho IB. De este orden se registraron organismos pertenecientes a la familia Gomphidae del Suborden Anisoptera, mientras que dentro del Suborden Zygoptera se registró una única morfoespecie. Aunque no se alcanzó el nivel de familia al identificar este último taxón, se consideró oportuno asignarle un valor, teniendo en cuenta la importancia de los odonatos como indicadores ecológicos. En la Tabla 1 se pueden consultar los valores asignados a cada taxón para el cálculo de los IB. Los IB se aplicaron a cada uno de los hábitats por separado y también a la suma de ambos hábitats. Se contabilizó la cantidad de taxones que cada IB incluyó, tanto por hábitat como para la suma de ambos hábitats.

\section{RESULTADOS}

Los parámetros físico-químicos mostraron niveles de OD cercanos al punto de saturación (S1: 7,46 mg/L OD, 81\% saturación; S2: 8,43 $\mathrm{mg} / \mathrm{L} \mathrm{OD}, 93 \%$ saturación), un $\mathrm{pH}$ con tendencia básica (S1: 8,66; S2: 8,49), la CE fue $783 \mu \mathrm{S} / \mathrm{cm}$ en $\mathrm{S} 1$ y $763 \mu \mathrm{S} / \mathrm{cm}$ en el S2, los SST fueron 8,59 $\mathrm{mg} / \mathrm{L}$ en $\mathrm{S} 1$ y $9,95 \mathrm{mg} / \mathrm{L}$ en $\mathrm{S} 2$, la $\mathrm{DBO}_{5}$ mostró valores de 3,17 mg/L en S1 y 3,41 mg/L en S2. La profundidad en los sitios de muestreo fue 70 $\mathrm{cm}$ en el $\mathrm{S} 1$ y $110 \mathrm{~cm}$ en el S2, la transparencia al disco de Secchi fue 70 y $75 \mathrm{~cm}$ respectivamente y la velocidad de la corriente en el S1 fue 0,35 $\mathrm{m} / \mathrm{s}$ mientras en el $\mathrm{S} 2$ se registró un área de remanso con un valor cercano a cero de velocidad de corriente. Los sedimentos fueron arenosos, en el $\mathrm{S} 1$ el $84 \%$ estuvo representado 
por las fracciones de arenas, mientras en el S2 lo estuvo el $72 \%$ de los sedimentos (Figura 2). En total se registraron 34 taxones. En S1, con

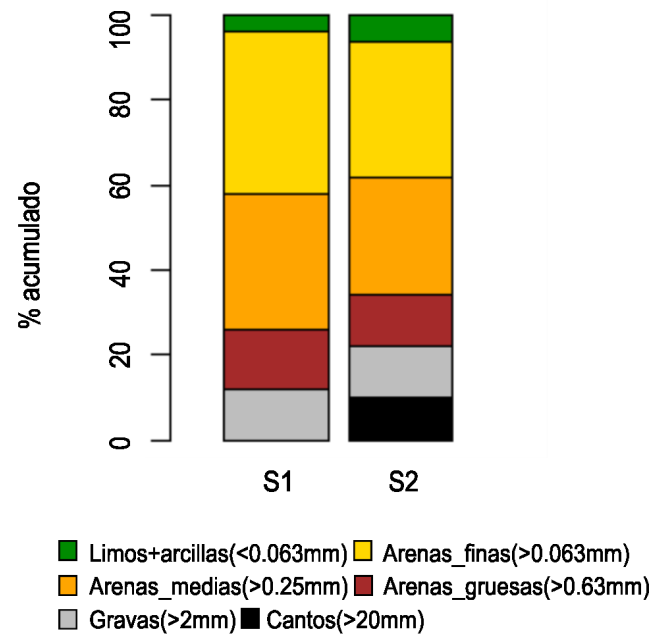

Figura 2. Granulometría de los sedimentos muestreados en cada sitio.

Figure 2. Granulometry of sediments in sampling sites.

un total de 30 taxones, se registraron 27 en macrófitas, indicando un $\mathrm{H}^{\prime}$ de 1,76. De este hábitat, 19 taxones fueron exclusivos. En los sedimentos se registraron 11 taxones, con un $\mathrm{H}^{\prime}$ de 1,23, y tres taxones fueron exclusivos de este hábitat. Ocho taxones fueron compartidos entre ambos hábitats. En el S2, con un total de 28 taxones, se registraron 26 en macrófitas, con un $\mathrm{H}^{\prime}$ de 1,27 y 15 taxones fueron exclusivos de este hábitat. En los sedimentos se registraron 13 taxones, con un $\mathrm{H}^{\prime}$ de 1,03 y teniendo solamente dos como exclusivos de este hábitat. Para el S2, 11 taxones fueron compartidos entre ambos hábitats. En la Tabla 1 se detalla la composición taxonómica de cada sitio y hábitat. En el S1 los Insecta dominaron en el hábitat de macrófitas, correspondiendo a este grupo el $43 \%$ de los organismos recolectados (Figura 3). En segundo término, se ubicaron los Oligochaeta con el $33 \%$ y los Crustacea con el 16\%. En el mismo sitio, el hábitat bentónico estuvo dominado por Hydridae, con el $48 \%$ de la abundancia total, le siguió Oligochaeta con el $27 \%$ y en tercer lugar Insecta con el 21\%. En el S2 fueron dominantes los Oligochaeta (Figura 3). En macrófitas se registraron como pertenecientes a este taxón el
$49 \%$ de los organismos recolectados, mientras el $38 \%$ pertenecieron a Insecta y el $12 \%$ a Crustacea. En los sedimentos, el $49 \%$ de los organismos perteneció a Oligochaeta, el 46\% a Insecta y le sigue Crustacea con sólo el $2 \%$. Para la clase Insecta, en todos los casos Diptera fue el orden más abundante y dentro de éste la familia Chironomidae. La aplicación de los IB mostró resultados muy diferentes entre los dos hábitats muestreados (Tablas 2 y 3). Para el cálculo realizado con los macroinvertebrados en macrófitas el resultado fue calidad de agua buena, sin contaminación; mientras el cálculo realizado con los macroinvertebrados de los sedimentos dio como resultado aguas contaminadas. La cantidad de taxones incluidos en el cálculo de los IB para el hábitat de macrófitas fue prácticamente el doble de los incluidos en el hábitat de sedimentos. De los taxones exclusivos en el hábitat de sedimentos, Hydropsichidae e Hirudinea aportaron su presencia al cálculo de los IB en el S1 e Hirudinea en el S2. El resto de los taxones incluidos en el cálculo estuvieron presentes en ambos hábitats. El resultado de los IB para los dos hábitats en conjunto no se modifica respecto a los valores calculados para el hábitat de macrófitas sólo. El IMRP por su parte incluyó el mayor número de taxones, respecto de los otros dos IB. Para su cálculo se incluyeron 25 taxones de los 30

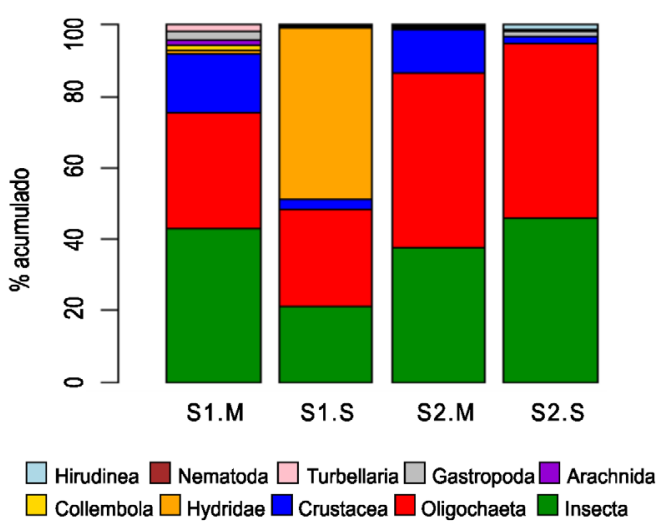

Figura 3. Abundancias proporcionales de los grupos taxonómicos registrados por cada sitio y hábitat muestreado. M: macrófitas; S: sedimentos.

Figure 3. Proportional abundances for taxonomic groups recorded, by site and habitat. M: macrophytes; S: sediments. 
Tabla 1. Lista de taxones registrados por hábitat (M: macrófitas; S: sedimentos) en los sitios de muestreo (S1: frente a la Reserva Natural Urbana Tara Inti, S2: aguas abajo de la ciudad de Termas de Río Hondo) y valor de sensibilidad asignado a cada uno para el cálculo de los IB aplicados.

Table 1. List of taxa recorded in each habitat (M: Macrophytes; S: Sediments) in the sampling sites (S1: in front of the Tara Inti Urban Natural Reserve, S2: downstream from Termas de Río Hondo city) and assigned sensitivity value of each one for the calculation of the applied IB.

\begin{tabular}{|c|c|c|c|c|c|c|c|}
\hline & S1-M & S2-M & S1-S & S2-S & IMRP & IBMWP & $\begin{array}{c}\text { BMWP' } \\
\text { Salí }\end{array}$ \\
\hline \multicolumn{8}{|l|}{ ARTHROPODA } \\
\hline \multicolumn{8}{|l|}{ INSECTA } \\
\hline \multicolumn{8}{|l|}{ Diptera } \\
\hline Chironomidae & $x$ & $x$ & $\mathrm{X}$ & $\mathrm{X}$ & 0,45 & 2 & 1 \\
\hline Ceratopogonidae & $x$ & $x$ & $X$ & $x$ & 0,4 & 4 & 4 \\
\hline Simuliidae & $x$ & $\mathrm{X}$ & & & 1,7 & 5 & 5 \\
\hline Stratiomyidae & $x$ & $x$ & & & 0,2 & 4 & 4 \\
\hline Muscidae & $x$ & $x$ & & & & & \\
\hline \multicolumn{8}{|l|}{ Ephemeroptera } \\
\hline Caenidae & $x$ & $x$ & $x$ & $x$ & 1,5 & 4 & 4 \\
\hline Baetidae & & $x$ & & $x$ & 1,5 & 4 & 4 \\
\hline Thysanoptera Thripidae & $x$ & $x$ & & & & & \\
\hline \multicolumn{8}{|l|}{ Trichoptera } \\
\hline Hydroptilidae & & $x$ & & $\mathrm{X}$ & 1,75 & 6 & 6 \\
\hline Hydropsychidae & & & $x$ & & 1,75 & 5 & 5 \\
\hline Lepidoptera Nepticulidae & & & $x$ & & & & \\
\hline \multicolumn{8}{|l|}{ Hemiptera } \\
\hline Corixidae & $x$ & & & & 0,8 & 3 & 3 \\
\hline Hebridae & $x$ & $x$ & & & 0,8 & & 3 \\
\hline Aphididae & $\mathrm{x}$ & $\mathrm{X}$ & & & & & \\
\hline \multicolumn{8}{|l|}{ Coleoptera } \\
\hline Dytiscidae & $x$ & $x$ & & & 0,35 & 3 & 3 \\
\hline Noteridae & $x$ & & & & & & \\
\hline Hydrophilidae & $x$ & $x$ & & & 0,35 & 3 & 3 \\
\hline Heteroceridae & $x$ & & & $x$ & & & \\
\hline \multicolumn{8}{|l|}{ Odonata } \\
\hline Zygoptera sp. & $x$ & $x$ & & & 1,4 & & 8 \\
\hline Gomphidae & $x$ & & & & 1,4 & 8 & 8 \\
\hline Orthoptera & $x$ & & & & & & \\
\hline ENTHOGNATA Collembola & $x$ & $x$ & & & 0,2 & & \\
\hline BRANCHIOPODA Cladocera & $x$ & $x$ & $x$ & $\mathrm{x}$ & 0,55 & & \\
\hline \multicolumn{8}{|l|}{ MALACOSTRACA Amphipoda } \\
\hline Hyalellidae & $x$ & $x$ & & & 0,9 & & \\
\hline MAXILLOPODA Copepoda & $x$ & $x$ & $\mathrm{X}$ & $\mathrm{X}$ & 0,35 & & 3 \\
\hline OSTRACODA & $x$ & & & & 0,4 & 3 & 3 \\
\hline \multicolumn{8}{|l|}{ ARACHNIDA } \\
\hline Trombidiformes & $x$ & $x$ & & & 0,3 & 4 & 4 \\
\hline \multicolumn{8}{|l|}{ Hydrachnidia } \\
\hline Araneae & & $\mathrm{X}$ & & & & & \\
\hline MOLLUSCAGastropoda & $x$ & $x$ & $\mathrm{X}$ & $x$ & 0,55 & 3 & \\
\hline PLATYHELMINTES Turbellaria & $x$ & $x$ & & $\mathrm{X}$ & & & \\
\hline \multicolumn{8}{|l|}{ ANNELLIDA } \\
\hline Oligochaeta & $x$ & $x$ & $x$ & $\mathrm{x}$ & 0,55 & 1 & 1 \\
\hline Hirudinea & & & $x$ & $\mathrm{X}$ & 0,55 & 3 & 3 \\
\hline CNIDARIA Hydrozoa & $x$ & $x$ & $x$ & & 1 & & \\
\hline \multicolumn{8}{|l|}{ Hydridae } \\
\hline NEMATODA & & $x$ & $\mathrm{x}$ & $\mathrm{x}$ & 0,1 & & \\
\hline
\end{tabular}

registrados, mientras para el IBMWP se incluyó solamente a 17 y para el BMWP'Salí se incluyó solo a 19. El ASPT se mostró consistente entre los diferentes hábitats, en todos los casos dio 
Tabla 2. Resultados de los índices bióticos aplicados a los invertebrados asociados a macrófitas. Referencias de color y clases de calidad: ver bibliografía citada para cada IB. $N^{\circ}$ T: número de taxones incluidos en el cálculo del IB. Calidad: clase de calidad del agua de acuerdo con el valor del IB.

Table 2. Results of biotic indices applied to macroinvertebrates that inhabitat macrophytes. Colors and water quality class references in bibliography cited for IB calculation. $\mathrm{N}^{\circ} \mathrm{T}$ : number of taxa included in the IB calculation. Calidad: water quality class according to IB value.

\begin{tabular}{|c|c|c|c|c|c|}
\hline M & ÍNDICE & & IBMWP' & $\begin{array}{l}\text { BMWP' } \\
\text { Salí }\end{array}$ & IMRP \\
\hline A & \multirow{4}{*}{ Sitio 1} & IB & 47 & 60 & 14,95 \\
\hline$C$ & & $\mathrm{~N}^{\circ} \mathrm{T}$ & 13 & 16 & \multirow{3}{*}{$\begin{array}{l}21 \\
\text { Contaminación } \\
\text { muy leve o nula }\end{array}$} \\
\hline $\begin{array}{l}\mathrm{R} \\
\mathrm{O}\end{array}$ & & Calidad & $\begin{array}{l}\text { Aguas } \\
\text { limpias }\end{array}$ & $\begin{array}{l}\text { Aguas } \\
\text { limpias }\end{array}$ & \\
\hline $\mathrm{F}$ & & ASPT & 3,62 & 3,75 & \\
\hline I & \multirow{4}{*}{ Sitio 2} & IB & 43 & 56 & 15,7 \\
\hline$T$ & & $\mathrm{~N}^{\circ} \mathrm{T}$ & 12 & 15 & 21 \\
\hline $\begin{array}{l}A \\
S\end{array}$ & & Calidad & $\begin{array}{l}\text { Aguas } \\
\text { limpias }\end{array}$ & $\begin{array}{l}\text { Aguas } \\
\text { limpias }\end{array}$ & \multirow[t]{2}{*}{$\begin{array}{l}\text { Contaminación } \\
\text { muy leve o nula }\end{array}$} \\
\hline & & ASPT & 3,58 & 3,73 & \\
\hline
\end{tabular}

como resultado un valor entre 3 y 4 , lo que corresponde a calidad del agua impactada. (Tabla 4).

Tabla 3. Detalle de los índices bióticos aplicados a los invertebrados asociados a sedimentos. Referencias de color y clases de calidad: ver bibliografía citada para cada IB. $N^{\circ}$ T: número de taxones incluidos en el cálculo del IB. Calidad: clase de calidad del agua de acuerdo con el valor del IB.

Table 3. Results of biotic indices apply to macroinvertebrates that inhabitat sediments. Colors and water quality class references in bibliography cited for IB calculation. $\mathrm{N}^{\circ} \mathrm{T}$ : number of taxa included in the IB calculation. Calidad: water quality class according to IB value.

\begin{tabular}{|c|c|c|c|c|c|}
\hline \multirow{3}{*}{$\begin{array}{l}S \\
E\end{array}$} & ÍNDICl & & IBMWP' & BMWP'Salí & IMRP \\
\hline & \multirow{4}{*}{ Sitio 1} & IB & 22 & 21 & 7,75 \\
\hline & & $\mathrm{N}^{\circ} \mathrm{T}$ & 6 & 7 & \multirow{3}{*}{$\begin{array}{l}11 \\
\text { Contaminación } \\
\text { débil }\end{array}$} \\
\hline I & & Calidad & $\begin{array}{l}\text { Aguas } \\
\text { contaminadas }\end{array}$ & $\begin{array}{l}\text { Aguas } \\
\text { contaminadas }\end{array}$ & \\
\hline 1 & & ASPT & 3,67 & 3,00 & \\
\hline $\mathrm{N}$ & \multirow{4}{*}{ Sitio 2} & IB & 27 & 26 & 8,25 \\
\hline TV & & $\mathrm{N}^{\circ} \mathrm{T}$ & 7 & 8 & \multirow{3}{*}{$\begin{array}{l}11 \\
\text { Contaminación } \\
\text { escasa }\end{array}$} \\
\hline o & & Calidad & $\begin{array}{l}\text { Aguas } \\
\text { contaminadas }\end{array}$ & $\begin{array}{l}\text { Aguas } \\
\text { contaminadas }\end{array}$ & \\
\hline & & ASPT & 3,86 & 3,25 & \\
\hline
\end{tabular}

\section{DISCUSIÓN}

Con los datos obtenidos se logró una primera aproximación al conocimiento de los ensambles de macroinvertebrados asociados a macrófitas en el río Dulce, así como a la aplicación de índices de calidad de agua basados en macroinvertebrados para este sistema fluvial. Tal como se registró en este estudio, otros sistemas fluviales de la región, como el río Paraná y el río Negro en la provincia del Chaco, presentan a Insecta, Oligochaeta y Crustacea como los grupos dominantes presentes en la vegetación acuática (Poi de Neiff, 2003). Entre los insectos, los grupos mejor representados fueron los dípteros, coleópteros y hemípteros, tal como reportan Poi de Neiffy Neiff (2006) para macrófitas de la planicie de inundación del río Paraná. Los órdenes Coleoptera y Hemiptera son reconocidos como representantes de la fauna asociada a macrófitas acuáticas en nuestro país (Bachman, 1998). De las familias de Coleoptera, Dityscidae es la que tiene mayor representación en los ecosistemas acuáticos a nivel mundial (Nilsson, 2001). Junto con Noteridae e Hidroptilidae fueron reportadas también por Torres et al. (2007) en estudios realizados sobre arroyos tributarios del río Uruguay, en sitios vegetados con Cortaderia sp., Eichhornia sp. y Myriophyllum sp. En cuanto a los hemípteros, estos autores reportan a Hebridae; aunque no registran a Corixidae para este tipo de ambientes. Varios de los taxones reportados son considerados exclusivos de hábitats terrestres. Los parches de macrófitas muestreados se desarrollan sobre los márgenes del curso de agua, por lo que es esperable que en este hábitat de transición entre la tierra y el agua se solape la presencia de especies con capacidad de aprovechar indistintamente los recursos de esta área marginal. Tanto Aphididae como Thripidae pueden alimentarse indistintamente de plantas terrestres o acuáticas, y Araneae puede aprovechar las macrófitas como hábitat aun sin presentar dependencia por el cuerpo de agua y, junto con Aphididae, son reportadas en el hábitat de macrófitas acuáticas en lagunas del área de Bañados del río Dulce (Naval et al., 2016). Orthoptera 
es un orden predominantemente terrestre, sin embargo, se reconoce que un pequeño porcentaje del mismo se desarrolla asociado a los parches de macrófitas, demostrando alta afinidad e inclusive dependencia reproductiva y alimentaria por estos hábitats acuáticos (Poi de Neif \& Neiff, 2006; Amédégnato \& Devriese, 2008; Capello et al., 2012). Oligochaeta Naididae junto con Insecta Chironomidae demostraron una alta dominancia en ambos hábitats. La importancia de estos grupos en el río Dulce ya había sido establecida en estudios sobre el hábitat bentónico (Leiva et al., 2017), en los cuales se reportan para el sistema fluvial varios géneros de quironómidos (Chironomus, Polypedilum, Tanytarsus y Pelomus, entre otros), y de oligoquetos (Pristina, Dero, Nais, Stylaria, Stephensoniana, entre otros), lo cual por otra parte resulta consistente con la prevalencia de sedimentos arenosos, con tamaños granulométricos medios y finos, en el lecho fluvial. aun cuando se tenga dificultad para identificar exactamente las familias, como en este caso una especie de Zygoptera. El IMRP por su parte también incluye a Collembola, Daphniidae, Copepoda, Turbellaria, Hydridae y Nematoda en el cálculo, taxones no considerados en los IB originados del BMWP'. Esto demuestra la importancia de utilizar en el monitoreo IB adaptados regionalmente, o de ajustarlos, para lograr resultados confiables (Crettaz et al., 2014). La aplicación de los IB diferenciando por hábitats resultó en evaluaciones muy diferentes de la calidad del agua. Al aplicar los IB a un hábitat en particular, se asumió el riesgo de perder el registro de taxones que no son afines a dicho hábitat, aun cuando estuvieran presentes en el ecosistema objeto del estudio. Pero es necesario considerar que, si esto se hiciera involuntariamente, por un error en el diseño de la investigación, podría derivar en resultados erróneos o por lo menos no consistentes con

Tabla 4. Detalle de los índices bióticos aplicados a los invertebrados asociados a ambos hábitats en conjunto. Referencias de color y clases de calidad: ver bibliografía citada para cada IB. $\mathrm{N}^{\circ} \mathrm{T}$ : número de taxones incluidos en el cálculo del IB. Calidad: clase de calidad del agua de acuerdo con el valor del IB.

Table 4. Results of biotic indices apply to macroinvertebrates that inhabitat both of habitats pooled. Colors and water quality class references in bibliography cited for IB calculation. $\mathrm{N}^{\circ} \mathrm{T}$ : number of taxa included in the IB calculation. Calidad: water quality class according to IB value.

\begin{tabular}{|c|c|c|c|c|c|}
\hline \multirow{9}{*}{ 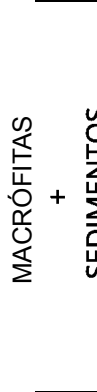 } & \multicolumn{2}{|c|}{ ÍNDICE } & \multirow{2}{*}{$\begin{array}{l}\text { IBMWP' } \\
55\end{array}$} & \multirow{2}{*}{$\begin{array}{l}\text { BMWP'Salí } \\
68\end{array}$} & \multirow{2}{*}{$\begin{array}{l}\text { IMRP } \\
17,35\end{array}$} \\
\hline & \multirow{4}{*}{ Sitio 1} & IB & & & \\
\hline & & $\mathrm{N}^{\circ} \mathrm{Tax}$ & 15 & 18 & \multirow{3}{*}{$\begin{array}{l}24 \\
\text { Contaminación muy leve o nula } \\
\text {... }\end{array}$} \\
\hline & & Calidad & Aguas limpias & Aguas limpias & \\
\hline & & ASPT & 3,67 & 3,78 & \\
\hline & \multirow{4}{*}{ Sitio 2} & IB & 46 & 59 & 16,25 \\
\hline & & $\mathrm{N}^{\circ} \mathrm{T}$ & 13 & 16 & \multirow{3}{*}{$\begin{array}{l}22 \\
\text { Contaminación muy leve o nula }\end{array}$} \\
\hline & & Calidad & Aguas limpias & Aguas limpias & \\
\hline & & ASPT & 3,54 & 3,69 & \\
\hline
\end{tabular}

De los IB aplicados, el BMWP' Salí y el IMRP resultaron en mayores valores de calidad de agua e incluyen a más familias en el cálculo, puesto que consideran a aquellas comunes en la región, como es el caso de Hyalellidae, Noteridae y Heteroceridae. Además, estos IB otorgan puntaje en forma general a familias de Odonata y Hemiptera, lo cual facilita la inclusión de los taxones registrados localmente, la realidad del sistema evaluado. EI ASPT, por otra parte, se mostró consistente entre los hábitats, reflejando que la mayoría de las familias registradas están asignadas a valores de sensibilidad moderados y bajos (Romero et al., 2011). Sin embargo, las variables físico-químicas medidas mostraron valores consistentes con una buena calidad del agua. El pH fue neutro, el valor de OD estuvo en un rango superior al 
$80 \%$ del nivel de saturación y la $\mathrm{DBO}_{5}$ registró valores muy bajos. La CE permite considerar a las aguas del río Dulce como de cierta salinidad, sin embargo, esto es consistente con la posición de este curso en el tramo inferior de una cuenca que drena formaciones litológicas ricas en minerales solubles. El tipo de hábitat muestreado influencia marcadamente la aplicación de IB para evaluar la calidad de aguas en el sistema fluvial del río Dulce. Sin dejar de lado la probable presencia de contaminantes específicos y el enriquecimiento de nutrientes en el sistema, que no fueron medidos, queda claro que muestrear sólo el hábitat de sedimentos derivó en una subestimación de la diversidad de macroinvertebrados para la aplicación de los IB. Cuando se agregó el hábitat de macrófitas, la diversidad de taxones registrados permitió establecer una buena calidad del agua, alcanzando el valor máximo de los índices. Resulta necesario por lo tanto identificar la diversidad de hábitats disponibles y su importancia relativa dentro del sistema fluvial para plantear la adecuada aplicación y el ajuste de herramientas de biomonitoreo como los IB. Finalmente, aunque a futuro se espera continuar con las investigaciones para proponer herramientas de monitoreo ajustadas a la fauna local, se concluye que de los IB aplicados en este trabajo tanto el BMWP adaptado para la cuenca del Salí como el IMRP podrían ser utilizados para el biomonitoreo en el río Dulce.

\section{AGRADECIMIENTOS}

Las autoras agradecen el apoyo para la realización de este trabajo al Concejo de Investigaciones Científicas y Tecnológicas de la Universidad Nacional de Santiago del Estero, CICYT UNSE, PID 23B/148 2013-2016; y al CONICET.

\section{REFERENCIAS}

Alba-Tercedor, J., y Sánchez-Ortega, A. (1988). Un método rápido y simple para evaluar la calidad biológica de las aguas corrientes basado en el de Hellawell (1978). Limnetica, 4: 51-56.
Amédégnato, C. \& Devriese, H. (2008). Global diversity of true and pygmy grasshoppers (Acridomorpha, Orthoptera) in freshwater. Hydrobiologia, 595 (1): 535-43.

APHA-AWWA-WPCF. (1989). Métodos normalizados para el análisis de aguas potables y residuales. Ediciones Díaz de Santos.

Armitage, P. D., Moss, D., Wright, J. F. \& Furse, M. T. (1983). The performance of a new biological water quality score system based on macroinvertebrates over a wide range of polluted running-water sites. Water Research,17 (3): 333-47.

Bachmann, A. O. (1998). Heteroptera acuáticos. En: Morrone, J. J. y Coscarón, S. (Eds.). Biodiversidad de artrópodos argentinos. Una perspectiva biotaxonómica (pp. 163-180). La Plata, Argentina: Ediciones Sur.

Barbour, M. T., Gerritsen, J., Snyder, B. D. \& Stribling J.B. (1999). Rapid bioassessment protocols for use in streams and wadeable rivers: Periphyton, benthic macroinvertebrates and fish. U.S. Environmental Protection Agency; Office of Water: Washington, D.C.

Bonada, N., Prat-Fornells, N., Resh, V. H. \& Statzner, B. (2006). Developments in aquatic insect biomonitoring: a comparative analysis of recent approaches. Annual Review of Entomology, 51(1): 495-523.

Brown, A. y Pacheco, S. (2006). Propuesta de actualización del mapa ecorregional de la Argentina. En: Brown, A., Martínez Ortiz, U., Acerbi, M. y Corcuera, J. (Eds.). La Situación Ambiental Argentina 2005 (pp 28-31). Recuperado en: http://siga.proyungas. org.ar/wp-content/uploads/2017/08/ PropuestaActualizacionEcoregiones.pdf Julio 7, 2018).

Burkart, R., Bárbaro, N. O., Sánchez, R. O. y Gómez, D. A. (1999). Eco-Regiones de la Argentina. Buenos Aires, Argentina: Administración de Parques Nacionales. Recuperado en: https://sib. gob.ar/archivos/Eco-Regiones_de_la_Argentina. pdf (Agosto 18, 2019).

Capello, S., Marchese, M. \& de Wysiecki, M. L. (2012). Feeding habits and trophic niche overlap of aquatic Orthoptera associated with macrophytes. Zoological Studies, 51(1): 51-58. 
Crettaz, M., Juárez, R., Aguer, I. y Borro, E. (2014). Aplicación de índices de calidad de agua en un arroyo pampeano utilizando macroinvertebrados bentónicos como bioindicadores. Biología Acuática, 30: 93-105.

Domínguez, E. y Fernández, H. R. (1998). Calidad de los ríos de la cuenca del Salí (Tucumán, Argentina) medida por un índice biótico. Serie Conservación de la Naturaleza, 12: 36-40.

Domínguez, E. y Fernández, H. R. (2009). Macroinvertebrados Bentónicos Sudamericanos. San Miguel de Tucumán. Argentina: Fundación Miguel Lillo.

Gee, G. W. \& Bauder, J. W. (1986). Particle-Size Analysis. In: Klute, A. (Ed.). Methods of Soil Analysis. Part 1: Physical and Mineralogical Methods (pp. 383-411). Wisconsin USA: Soil Science Society of America, American Society of Agronomy.

Hamada, N., Nessimian, J. L. \& Querino, R. B. (2014). Insetos aquáticos na Amazônia brasileira: taxonomia, biologia e ecologia. 1st ed. Manaos, Brasil: Embrapa Meio Norte Livros Científicos.

Hammer, Ø., Harper, D.A.T. \& Ryan, P.D. (2018). PAST Version 3.2. Paleontological Statistics Software Package. University of Oslo. Recuperado en: https://folk.uio.no/ohammer/past/. (fecha de acceso: 15-06-2020)

Hanson, P., Springer, M. y Ramirez, A. (2010). Introducción a los Grupos de Macroinvertebrados Acuáticos. Revista de Biologia Tropical, 58 (Suppl. 4): 3-37.

Jáimez-Cuéllar, P., Vivas, S., Bonada, N., Robles, S., Mellado, A., Álvarez, M., Avilés, J., Casas, J. Ortega, M., Isabel Pardo, Prat, N., Rieradevall, M., Sáinz-Cantero, C. E., Sánchez-Ortega, A., Suárez, M. L., Toro, M., Vidal-Abarca, M. Rosario, Zamora-Muñoz, C. y Alba-Tercedor, J. (2002). Protocolo GUADALMED (PRECE). Limnetica, 21(4): 187-204.

Leiva, M., Marchese, M., Diodato, L. \& Tévez, H. (2017). Assessing disruption of longitudinal connectivity on macroinvertebrate assemblages in a semiarid lowland river. Acta Limnologica Brasiliensia, 29: e19.
Macchi, P. A. (2016). Macroinvertebrados acuáticos como indicadores ecológicos de cambios en el uso del suelo en mallines del sudoeste de la provincia de Río Negro. (Tesis doctoral N 1473, Facultad de Ciencias Naturales y Museo, Universidad Nacional de La Plata).

Naval, C., Benítez, E. M., Abregú, D., Fernández, G., Perea, V., Harguinteguy, C. y Torres, R. (2016). Estudio de las comunidades de macroinvertebrados asociados a macrófitas acuáticas de la planicie de inundación del río Utis (Santiago del Estero, Argentina). VI Reunión Binacional de Ecología. pp. 282. Lugar: Puerto Iguazú

Poi de Neiff, A. (2003). Limnología del Iberá: aspectos físicos, químicos y biológicos de las aguas. Corrientes, Argentina: Editorial de la Universidad Nacional del Nordeste.

Nilsson, A. N. (2001). World Catalogue of Insects, Volume 3: Dystiscidae (Coleoptera). Steenstrup, Denmark: Apollo Books.

Poi De Neiff, A. y Neiff, J. J. (2006). Riqueza de especies y similaridad de los invertebrados que viven en plantas flotantes de la planicie de inundación del río Paraná, Argentina. Interciencia, 31(3): 220-25.

Prat-Fornells, N., Ríos, B., Acosta, R. y Rieradevall, M. (2009). Los macroinvertebrados como indicadores de calidad de las aguas. En: Domínguez, E. y Fernández, H. R. (Eds.). Macroinvertebrados bentónicos sudamericanos (pp. 631-654). San Miguel de Tucumán. Argentina: Fundación Miguel Lillo.

Ramírez, A. (2010). Métodos de recolección. Revista de Biología Tropical, 5858 (Suppl. 4): 41-50.

Reid, A. J. (2019). Emerging threats and persistent conservation challenges for freshwater biodiversity. Biological Reviews, 94 (3): 849-73.

Rodrigues Capítulo, A., Ocón, C. S. y Tangorra, M. (2003). Una visión bentónica de arroyos y río pampeanos. Biología Acuática 21: 1-18.

Roldán-Pérez, G. (2016). Los macroinvertebrados como bioindicadores de la calidad del agua: cuatro décadas de desarrollo en Colombia 
y Latinoamerica. Revista de la Academia Colombiana de Ciencias Exactas, Físicas y Naturales, 40 (155): 254-274.

Romero, F. (2011). Estudio integral de la cuenca del Río Lules (Tucumán): Aspectos biológicos. En: Fernández, H. R. y Barber, H. (Eds.). La cuenca del río Lules: una aproximación multidisciplinaria a su complejidad (pp. 110-135). Tucumán, Argentina: Editorial de la Universidad Nacional de Tucumán.
Segnini, S. (2003). El uso de macroinvertebrados béntonicos como indicadores de la condición ecológica de los cuerpos de agua corriente. Ecotropicos, 16 (2): 45-63.

Torres, P. L. M., Mazzucconi, S. A. y Michat, M. C. (2007). Los Coleópteros y Heterópteros acuáticos del Parque Nacional El Palmar (Provincia de Entre Ríos, Argentina): Lista faunística, diversidad y distribución. Revista de la Sociedad Entomológica Argentina, 66 (3-4): 127-54. 